\title{
EXISTENCE RESULTS FOR ABSTRACT DEGENERATE NEUTRAL FUNCTIONAL DIFFERENTIAL EQUATIONS
}

\author{
EDUARDO HERNÁNDEZ ${ }^{\bowtie}$ and KRISHNAN BALACHANDRAN
}

(Received 12 August 2009)

Abstract

In this paper we discuss the existence of solutions for a class of abstract degenerate neutral functional differential equations. Some applications to partial differential equations are considered.

2000 Mathematics subject classification: primary 35R10, 34K40; secondary 34K30.

Keywords and phrases: neutral equations, mild solutions, semigroup of bounded linear operators.

\section{Introduction}

In this paper we study the existence of solutions for a class of abstract neutral functional differential equations of the form

$$
\begin{gathered}
\frac{d}{d t}\left[x(t)+D(t) x\left(t-r_{1}\right)\right]=A x(t)+f\left(t, x_{t}\right), \quad t \in[0, a], \\
x_{0}=\varphi \in \mathcal{C}, \quad \mathcal{C}=C([-r, 0] ; X),
\end{gathered}
$$

where $A: D(A) \subset X \rightarrow X$ is the infinitesimal generator of a $C_{0}$-semigroup of bounded linear operators $(T(t))_{t \geq 0}$ on a Banach space $(X,\|\cdot\|),(D(t))_{t \in[0, a]}$ is a family of closed linear operators defined on a common domain $\mathcal{D} \neq X, 0<r_{1} \leq r$ and $f:[0, a] \times \mathcal{C} \rightarrow X$ is a suitable function.

In this work we continue the developments in Hernández and O'Regan [18] on the existence of solutions for abstract neutral systems. The basic novelty with respect to [18] and the general literature on neutral equations is the fact that the operators $D(t)$ can be unbounded.

In order to review the associated literature on neutral equations and understand the motivations for this work, it is convenient to introduce the following more general model of a neutral system:

$$
\begin{gathered}
\frac{d}{d t}\left[x(t)+g\left(t, x_{t}\right)\right]=A x(t)+f\left(t, x_{t}\right), \quad t \in[0, a], \\
x_{0}=\varphi \in \mathcal{C} .
\end{gathered}
$$

(C) 2010 Australian Mathematical Publishing Association Inc. 0004-9727/2010 \$16.00 
Datko [9] and Adimy and Ezzinbi [1] studied some linear neutral systems similar to (1.3)-(1.4) under the strong assumption that the function $g(\cdot)$ is $D(A)$-valued continuous. If $A$ is the generator of a $C_{0}$-semigroup of bounded linear operators $(T(t))_{t \geq 0}$ (the case studied by Datko), this assumption arises from the associated integral equation

$$
\begin{gathered}
u(t)=T(t)[\varphi(0)+g(0, \varphi)]-g\left(t, u_{t}\right)-\int_{0}^{t} A T(t-s) g\left(s, u_{s}\right) d s \\
+\int_{0}^{t} T(t-s) f\left(s, u_{s}\right) d s, \quad t \in[0, a]
\end{gathered}
$$

since except in trivial cases, the operator function $A T(\cdot)$ is not integrable in the operator topology on $[0, b]$, for $b>0$. The same reason explains the use of this assumption in [1], where the case in which $A$ is a Hille-Yosida type operator is studied.

In the papers [16-18] the system (1.3)-(1.4) was studied assuming that $A$ is the generator of an analytic semigroup $(T(t))_{t \geq 0}$ and under a more general and less restrictive assumption on $g(\cdot)$, which is a particular case of the following condition.

$\left(\mathrm{H}_{\mathrm{g}}\right)$ There exists a Banach space $\left(Y,\|\cdot\|_{Y}\right)$ continuously embedded in $X$ and a function $H \in L^{1}([0, a])$ such that $g \in C([0, a] \times \mathcal{C} ; Y)$ and $\|A T(t)\|_{\mathcal{L}(Y, X)} \leq$ $H(t)$ for all $t \in[0, a]$.

The condition $\left(\mathrm{H}_{\mathrm{g}}\right)$ is satisfied in several situations, for example, the case in which $Y$ is an interpolation space between $X$ and $D(A)$. However, it remains an important restriction on the system.

In the papers [2-5, 10] (among an extensive literature) an alternative assumption has been used to treat some neutral equations. In these it is assumed that $A$ is the generator of a compact $C_{0}$-semigroup $(T(t))_{t \geq 0}$ and the set $\{A T(t): t \in(0, b]\}$ is bounded in the operator topology. However, as has been pointed out in [16], these conditions are valid if and only if $A$ is bounded and $\operatorname{dim} X<\infty$, which restricts the applications to ordinary differential equations. Moreover, if the compactness assumption is removed, it follows that $A$ is bounded, which remains a strong restriction.

On the other hand, in the recent paper [18] local existence results are established for a neutral system with mixed delay similar to (1.1)-(1.2) without using the above restrictions. Our purpose in this paper is to continue the developments in [18] by considering the case in which the operators $D(t)$ are unbounded. We will refer to systems of this type as abstract degenerate neutral differential systems.

Neutral differential equations arise in many areas. The literature on ordinary neutral functional differential equations is very extensive and we refer the reader to the book by Hale and Verduyn Lunel [15] for details.

Partial differential neutral equations arise, for instance, in the theory of heat conduction in fading memory materials. In the classical theory of heat conduction, it is assumed that the internal energy and the heat flux depend linearly on the temperature $u(\cdot)$ and on its gradient $\nabla u(\cdot)$. Under these conditions, the classical heat equation describes sufficiently well the evolution of the temperature in different 
types of materials. However, this description is not satisfactory in materials with fading memory. In the theory developed in [14, 23], the internal energy and the heat flux are described as functionals of $u(\cdot)$ and $u_{x}(\cdot)$. The following equation (see, for example, $[6-8,22])$, has frequently been used to describe this phenomenon:

$$
\begin{gathered}
\frac{\partial}{\partial t}\left[u(t, x)+\int_{-\infty}^{t} k_{1}(t-s) u(s, x) d s\right]=c \Delta u(t, x)+\int_{-\infty}^{t} k_{2}(t-s) \Delta u(s, x) d s, \\
u(t, x)=0, \quad x \in \partial \Omega .
\end{gathered}
$$

In this equation, $\Omega \subset \mathbb{R}^{n}$ is open, bounded and has smooth boundary, $(t, x) \in$ $[0, \infty) \times \Omega, u(t, x)$ represents the temperature in $x$ at time $t, c$ is a physical constant and $k_{i}: \mathbb{R} \rightarrow \mathbb{R}, i=1,2$, are the internal energy and the heat flux relaxation, respectively. By assuming that the solution $u(\cdot)$ is known on $(-\infty, 0]$ and $k_{2} \equiv 0$, we can represent this equation in the abstract form (1.3)-(1.4).

We also find partial neutral differential systems in the theory of population dynamics. There exists an extensive literature on ordinary neutral differential equations; see, for instance, $[11,12,20,21]$ and the references therein. Looking at these papers, it is natural to think that the abstract equation (2.1)-(2.2) can be used to consider spatial diffusion phenomena, which arise because of the natural tendency of biological populations to migrate from high population density regions to regions with low density.

We now introduce some basic concepts, definitions and results. In the rest of this paper, $A: D(A) \subseteq X \rightarrow X$ is the generator of an analytic semigroup of bounded linear operators $(T(t))_{t \geq 0}$ on a Banach space $(X,\|\cdot\|),(D(t))_{t \geq 0}$ is a one-parameter family of closed linear operators defined on a common domain $\mathcal{D} \subset D(A), 0<r_{1} \leq r$, $\mathcal{C}$ is the space $C([-r, 0] ; X)$ endowed with the uniform norm denoted by $\|\cdot\|_{\mathcal{C}}$ and $f:[0, a] \times \mathcal{C} \rightarrow X$ is a continuous function. The notation $[D(A)]$ stands for the space $D(A)$ endowed with the graph norm and $M>0$ is such that $\|T(t)\| \leq M$ for all $t \in[0, a]$.

For simplicity, we assume that $(T(t))_{t \geq 0}$ is uniformly bounded and $0 \in \rho(A)$. In this case, it is possible to define the fractional power $(-A)^{\alpha}, 0<\alpha \leq 1$, as a closed linear operator on its domain $D\left((-A)^{\alpha}\right)$. Furthermore, $D\left((-A)^{\alpha}\right)$ is dense in $X$ and the expression $\|x\|_{\alpha}:=\left\|(-A)^{\alpha} x\right\|, x \in D\left((-A)^{\alpha}\right)$, defines a norm on $D\left((-A)^{\alpha}\right)$. Henceforth we denote by $X_{\alpha}$ the space $D\left((-A)^{\alpha}\right)$ with the norm $\|\cdot\|_{\alpha}$. From [24] we note the following properties.

\section{LEMMA 1.1. The following conditions are satisfied.}

(a) Let $0<\alpha \leq 1$. Then $X_{\alpha}$ is a Banach space.

(b) If $0<\beta<\alpha \leq 1$ then $X_{\alpha} \hookrightarrow X_{\beta}$ and the embedding is compact when the resolvent operator of $A$ is compact.

(c) For each $\gamma \in(0,1)$, there exists $C_{\gamma}>0$ such that $\left\|(-A)^{\gamma} T(t) x\right\| \leq C_{\gamma} / t^{\gamma}\|x\|$ for all $t \in(0, a]$ and every $x \in X$.

Let $\left(Z,\|\cdot\|_{Z}\right)$ and $\left(W,\|\cdot\|_{W}\right)$ be Banach spaces. Throughout this paper, let $\left(\mathcal{L}(Z, W),\|\cdot\|_{Z, W}\right)$ denote the space formed by all the bounded linear operators 
from $Z$ into $W$ endowed with the uniform operator norm $\|\cdot\|_{\mathcal{L}(Z, W)}$. We write simply $\left(\mathcal{L}(Z),\|\cdot\|_{\mathcal{L}(Z)}\right)$ when $Z=W$. Let $B_{r}(z, Z)$ represent the closed ball with center at $z$ and radius $r$ in $Z$.

\section{Existence of solutions}

In this section we discuss the existence of solutions for neutral equations of the form

$$
\begin{gathered}
\frac{d}{d t}\left[x(t)+D(t) x\left(t-r_{1}\right)\right]=A x(t)+f\left(t, x_{t}\right), \quad t \in[\sigma, \sigma+b], \\
x_{\sigma}=\varphi \in C([-r, 0] ; X) .
\end{gathered}
$$

To deal with (2.1)-(2.2), we introduce and study two different kinds of solution (mild and $S$-mild solution) which arise from different regularity-type conditions on the functions $\varphi$ and $t \rightarrow D(t) \varphi\left(t-r_{1}\right)$.

REMARK 2.1. In the rest of this work, $g:\left[0, r_{1}\right] \rightarrow X$ is the function defined by $g(t)=D(t) \varphi\left(t-r_{1}\right)$ and $y:[-r, a] \rightarrow X$ is given by $y_{0}=\varphi$ and $y(t)=T(t) \varphi(0)$ for $t \in[0, a]$.

2.1. Existence of mild solutions. Motivated by [18], we introduce the first type of solution for (2.1)-(2.2).

Definition 2.2. A function $u \in C([-r+\sigma, \sigma+b] ; X), b>0, \sigma \in \mathbb{R}$, is called a mild solution of the neutral equation (2.1)-(2.2) if $u_{\sigma}=\varphi$ and

$$
\begin{aligned}
u(t)=T(t-\sigma)\left[\varphi(0)+D(0) \varphi\left(-r_{1}\right)\right]-D(t) u\left(t-r_{1}\right) & \\
& -\int_{\sigma}^{t} A T(t-s) D(s) u\left(s-r_{1}\right) d s+\int_{\sigma}^{t} T(t-s) f\left(s, u_{s}\right) d s,
\end{aligned}
$$

for all $t \in[\sigma, \sigma+b]$.

We now establish our first existence result.

THEOREM 2.3. Assume that the following conditions are satisfied.

(a) There exist $\alpha \in(0,1)$ and a Banach space $\left(Y,\|\cdot\|_{Y}\right) \hookrightarrow(X,\|\cdot\|)$ such that $D(\cdot) \in C\left([0, a], \mathcal{L}\left(X_{\alpha}, Y\right)\right)$ and $A T(\cdot) \in L^{1}([0, a], \mathcal{L}(Y, X))$.

(b) There exits $0<\mu<1-\alpha$ such that $\varphi(0) \in X_{\alpha+\mu}$ and $g \in C\left(\left[0, r_{1}\right], X_{\alpha+\mu}\right)$.

(c) The function $f(\cdot)$ is continuous and there exists $L_{f}>0$ such that

$$
\left\|f\left(t, \psi_{1}\right)-f\left(t, \psi_{2}\right)\right\| \leq L_{f}\left\|\psi_{1}-\psi_{2}\right\|_{\mathcal{C}}, \quad t \in[0, a], \psi_{i} \in \mathcal{C} .
$$

Then there exists a unique mild solution of (1.1)-(1.2) on [-r, 2r $\left.r_{1}\right]$. 
PRoOF. Let $\Gamma_{1}: C\left(\left[-r, r_{1}\right] ; X\right) \rightarrow C\left(\left[-r, r_{1}\right] ; X\right)$ be the map defined by $(\Gamma u)_{0}=\varphi$ and

$$
\begin{gathered}
\Gamma_{1} u(t)=T(t)[\varphi(0)+g(0)]-g(t)-\int_{0}^{t} A T(t-s) g(s) d s \\
+\int_{0}^{t} T(t-s) f\left(s, u_{s}\right) d s, \quad t \in\left[0, r_{1}\right] .
\end{gathered}
$$

From Bochner's theorem on integrable functions and the estimate

$$
\begin{aligned}
\int_{0}^{t}\|A T(t-s) g(s)\| d s & \leq \int_{0}^{t}\left\|(-A)^{1-(\alpha+\mu)} T(t-s)(-A)^{\alpha+\mu} g(s)\right\| d s \\
& \leq\|g\|_{C\left(\left[0, r_{1}\right], X_{\alpha+\mu}\right)} \int_{0}^{t} \frac{C_{1-(\alpha+\mu)}}{(t-s)^{1-(\alpha+\mu)}} d s \\
& \leq\|g\|_{C\left(\left[0, r_{1}\right], X_{\alpha+\mu}\right)} \frac{r_{1}^{\alpha+\mu}}{\alpha+\mu},
\end{aligned}
$$

we infer that the function $s \rightarrow A T(t-s) g(s)$ is integrable on $[0, t]$ for all $t \in\left[0, r_{1}\right]$ and $\Gamma_{1} u \in C\left(\left[-r, r_{1}\right] ; X\right)$ for all $u \in C\left(\left[-r, r_{1}\right] ; X\right)$. Moreover, from the inequality

$$
\sup _{\theta \in[0, t]}\left\|\Gamma_{1}^{k} u(\theta)-\Gamma_{1}^{k} v(\theta)\right\| \leq \frac{\left(M L_{f}\right)^{k}}{k !} \int_{0}^{t} \sup _{\theta \in[0, s]}\|u(\theta)-v(\theta)\| d s, \quad t \in\left[0, r_{1}\right],
$$

it follows that $\Gamma_{1}^{k}(\cdot)$ is a contraction for $k$ large enough and there exits a unique fixed point $u^{1}(\cdot)$ of $\Gamma_{1}(\cdot)$. Obviously, $u^{1}(\cdot)$ is a mild solution of (1.1)-(1.2) on $\left[-r, r_{1}\right]$.

Next, we show that $u^{1} \in C\left(\left[0, r_{1}\right] ; X_{\alpha}\right)$. Since $\varphi(0) \in X_{\alpha}$ and the semigroup $(T(t))_{\geq 0}$ is analytic, it follows that $v(t)=T(t)\left[\varphi(0)+D(0) \varphi\left(-r_{1}\right)\right]-D(t)$ $\varphi\left(t-r_{1}\right) \in X_{\alpha}$ for all $t \in\left[0, r_{1}\right]$. Moreover, from the estimate

$$
\begin{aligned}
\|(-A)^{\alpha} & \left(u^{1}(t)-v(t)\right) \| \\
\leq & \int_{0}^{t}\left\|A^{1-\mu} T(t-s)\right\|\left\|(-A)^{\alpha+\mu} g(s)\right\| d s \\
& +\int_{0}^{t}\left\|(-A)^{\alpha} T(t-s)\right\|\left\|f\left(s, u_{s}^{1}\right)\right\| d s \\
\leq & \|g\|_{C\left(\left[0, r_{1}\right], X_{\alpha+\mu}\right)} \int_{0}^{t} \frac{C_{1-\mu}}{(t-s)^{1-\mu}} d s+\sup _{s \in\left[0, r_{1}\right]}\left\|f\left(s, u_{s}^{1}\right)\right\| \int_{0}^{t} \frac{C_{\alpha}}{(t-s)^{\alpha}} d s \\
\leq & \left(\|g\|_{C\left(\left[0, r_{1}\right], X_{\alpha+\mu}\right)}+\sup _{s \in\left[0, r_{1}\right]}\left\|f\left(s, u_{s}^{1}\right)\right\|\right)\left(\frac{C_{1-\mu} r_{1}^{\mu}}{\mu}+\frac{C_{\alpha} r_{1}^{1-\alpha}}{1-\alpha}\right)
\end{aligned}
$$

we conclude that $u^{1}(t) \in X_{\alpha}$ and $u^{1} \in C\left(\left[-r, r_{1}\right] ; X_{\alpha}\right)$ since $(-A)^{\alpha}$ is a closed operator. Moreover, from this fact it follows that the function $s \rightarrow D(s) u^{1}\left(s-r_{1}\right)$ belongs to $C\left(\left[r_{1}, 2 r_{1}\right] ; Y\right)$. 
We now consider the neutral equation

$$
\begin{aligned}
\frac{d}{d t}\left[x(t)+D(t) x\left(t-r_{1}\right)\right] & =A x(t)+f\left(t, x_{t}\right), \quad t \in\left[r_{1}, 2 r_{1}\right], \\
x_{r_{1}} & =\left(u^{1}\right)_{r_{1}} .
\end{aligned}
$$

Let $\Gamma_{2}: C\left(\left[r_{1}-r, 2 r_{1}\right] ; X\right) \rightarrow C\left(\left[r_{1}-r, 2 r_{1}\right] ; X\right)$ be defined by $\left(\Gamma_{2} u\right)_{r_{1}}=\left(u^{1}\right)_{r_{1}}$ and

$$
\begin{aligned}
\Gamma_{2} u(t)=T(t & \left.-r_{1}\right)\left[u^{1}\left(r_{1}\right)+D\left(r_{1}\right) u^{1}\left(-r_{1}\right)\right]-D(t) u^{1}\left(t-r_{1}\right) \\
& -\int_{r_{1}}^{t} A T(t-s) D(s) u^{1}\left(s-r_{1}\right) d s+\int_{r_{1}}^{t} T(t-s) f\left(s, u_{s}\right) d s
\end{aligned}
$$

for $t \in\left[r_{1}, 2 r_{1}\right]$. Remarking that $s \rightarrow D(s) u^{1}\left(s-r_{1}\right) \in C\left(\left[r_{1}, 2 r_{1}\right] ; Y\right)$, from the estimate

$$
\begin{aligned}
& \int_{r_{1}}^{t} \| A T(t-s) D(s) u^{1}\left(s-r_{1}\right) \| d s \\
& \quad \leq \int_{r_{1}}^{t}\|A T(t-s)\|_{\mathcal{L}(Y, X)}\left\|D(s) u^{1}\left(s-r_{1}\right)\right\|_{Y} d s \\
& \quad \leq \sup _{s \in\left[0, r_{1}\right]}\left\|D(s) u^{1}\left(s-r_{1}\right)\right\|_{Y}\|A T(\cdot)\|_{L^{1}\left(\left[0, r_{1}\right], \mathcal{L}(Y, X)\right)}
\end{aligned}
$$

we infer that $\Gamma_{2} u \in C\left(\left[r_{1}-r, 2 r_{1}\right] ; X\right)$ for every $u \in C\left(\left[r_{1}-r, 2 r_{1}\right] ; X\right)$. Proceeding now as in the first part of the proof, we can show that $\Gamma_{2}^{k}(\cdot)$ is a contraction for $k$ large and that there exists a unique fixed point $u^{2} \in C\left(\left[r_{1}-r, 2 r_{1}\right] ; X\right)$ of $\Gamma_{2}(\cdot)$.

To finish, we note that the function $u:\left[-r, 2 r_{1}\right] \rightarrow X$ defined by $u(t)=u^{1}(t)$ for $t \in\left[-r, r_{1}\right]$ and $u(t)=u^{2}(t)$ for $t \in\left[r_{1}, 2 r_{1}\right]$ is a mild solution of (1.1)-(1.2) on $\left[-r, 2 r_{1}\right]$. The proof is now complete.

In the next result, we establish the existence of a solution using a fixed point criterion for completely continuous maps.

THEOREM 2.4. Assume that conditions $(a)$ and $(b)$ of Theorem 2.3 hold and that the semigroup $(T(t))_{t \geq 0}$ is compact. Suppose, in addition, that $f \in C([0, a] \times \mathcal{C}, X)$ and that there are $m \in C([0, a] ;[0, \infty))$ and a nondecreasing function $W:[0, \infty) \rightarrow$ $(0, \infty)$ such that $\|f(t, \psi)\| \leq m(t) W\left(\|\psi\|_{\mathcal{C}}\right)$ for every $(t, \psi) \in[0, a] \times \mathcal{C}$. If

$$
M \int_{0}^{r_{1}} m(s) d s<\int_{C(\varphi)}^{\infty} \frac{d s}{W(s)},
$$

where $C(\varphi)=(M+1)\left(\|\varphi\|+\|g\|_{C\left(\left[0, r_{1}\right] ; X\right)}\right)+C_{1-(\alpha+\mu)}\|g\|_{C\left(\left[0, r_{1}\right] ; X_{\alpha+\mu}\right)} r_{1}^{\alpha+\mu} / \alpha+\mu$, then there exists a mild solution of (1.1)-(1.2) on $\left[-r_{1}, b\right]$ for some $r_{1}<b \leq a$.

PROOF. Let $\Gamma_{1}(\cdot)$ be the map defined in the proof of Theorem 2.3. From the proof of Theorem 2.3 we know that $\Gamma_{1}(\cdot)$ is well defined. Moreover, a standard argument using 
the Lebesque dominated convergence theorem and the Ascoli-Arzelà criterion allows us to prove that $\Gamma_{1}(\cdot)$ is completely continuous.

In order to apply the Leray-Schauder alternative theorem [13, Theorem 6.5.4], we obtain a priori estimates for the solutions of the integral equation $z=\lambda \Gamma_{1} z, \lambda \in(0,1)$. Let $\lambda \in(0,1), u^{\lambda}(\cdot)$ be a solution of $z=\lambda \Gamma_{1} z$ and $\alpha^{\lambda}:\left[0, r_{1}\right] \rightarrow \mathbb{R}$ be the function defined by $\alpha^{\lambda}(t)=\|\varphi\|_{\mathcal{C}}+\sup _{\theta \in[0, t]}\left\|u^{\lambda}(\theta)\right\|$. Then, for $t \in\left[0, r_{1}\right]$ we obtain

$$
\begin{aligned}
\left\|u^{\lambda}(t)\right\| \leq M\|\varphi(0)\|+(M+1)\|g\|_{C\left(\left[0, r_{1}\right] ; X\right)} & +\|g\|_{C\left(\left[0, r_{1}\right] ; X_{\alpha+\mu}\right)} \int_{0}^{t} \frac{C_{1-(\alpha+\mu)}}{(t-s)^{1-(\alpha+\mu)}} d s+M \int_{0}^{t} m(s) W\left(\left\|u_{s}\right\|_{\mathcal{C}}\right) d s \\
\leq M\|\varphi(0)\|+(M+1)\|g\|_{C\left(\left[0, r_{1}\right] ; X\right)} & \quad+C_{1-(\alpha+\mu)}\|g\|_{C\left(\left[0, r_{1}\right] ; X_{\alpha+\mu}\right)} \frac{r_{1}^{\alpha+\mu}}{\alpha+\mu}+M \int_{0}^{t} m(s) W\left(\alpha^{\lambda}(s)\right) d s,
\end{aligned}
$$

and hence,

$$
\alpha^{\lambda}(t) \leq C(\varphi)+M \int_{0}^{t} m(s) W\left(\alpha^{\lambda}(s)\right) d s
$$

If we denote the left-hand side of (2.5) by $\beta_{\lambda}(t)$, then $\beta_{\lambda}^{\prime}(t) \leq M m(t) W\left(\beta_{\lambda}(t)\right)$ and

$$
\int_{C(\varphi)}^{\beta_{\lambda}(t)} \frac{d s}{W(s)} \leq M \int_{0}^{r_{1}} m(s) d s<\int_{C(\varphi)}^{\infty} \frac{d s}{W(s)} d s
$$

which permit the assertion that $\left\{\beta_{\lambda}: \lambda \in(0,1)\right\}$ is bounded in $C\left(\left[0, r_{1}\right]\right)$, and, as a consequence, that $\left\{z^{\lambda}: \lambda \in(0,1)\right\}$ is bounded in $C\left(\left[-r, r_{1}\right] ; X\right)$.

From the above remarks and [13, Theorem 6.5.4], there exists a fixed point $u^{1} \in C\left(\left[-r, r_{1}\right] ; X\right)$ of $\Gamma_{1}$. Moreover, the same argument as used in the proof of Theorem 2.3 allows us to show that $u^{1} \in C\left(\left[0, r_{1}\right] ; X_{\alpha}\right), s \rightarrow D(s) u^{1}$ $\left(s-r_{1}\right) \in C\left(\left[r_{1}, 2 r_{1}\right] ; Y\right)$ and the function $s \rightarrow A T(t-s) D(s) u^{1}\left(s-r^{1}\right)$ belongs to $L^{1}\left(\left[r_{1}, t\right] ; X\right)$ for all $t \in\left[r_{1}, 2 r_{1}\right]$.

Let $r_{1}<b \leq 2 r_{1}$ be such that

$$
M \int_{r_{1}}^{b} m(s) d s<\int_{C\left(u^{1}\right)}^{\infty} \frac{d s}{W(s)}
$$

where

$$
\begin{aligned}
C\left(u^{1}\right)=(M+1) & \left(\left\|u_{r_{1}}^{1}\right\|_{\mathcal{C}}+\sup _{s \in\left[0, r_{1}\right]}\left\|D(s) u^{1}\left(s-r_{1}\right)\right\|\right) \\
& +\sup _{\theta \in\left[0, r_{1}\right]}\left\|D(\theta) u^{1}\left(\theta-r_{1}\right)\right\|_{Y} \int_{r_{1}}^{b}\|A T(s)\|_{\mathcal{L}(Y, X)} d s .
\end{aligned}
$$


Arguing as in the above steps, we can show that the map $\Gamma_{2}: C\left(\left[r_{1}-r, 2 r_{1}\right] ; X\right) \rightarrow$ $C\left(\left[r_{1}-r, 2 r_{1}\right] ; X\right)$ given by $\left(\Gamma_{2} u\right)_{r_{1}}=\left(u^{1}\right)_{r_{1}}$ and

$$
\begin{aligned}
\Gamma_{2} u(t)=T(t & \left.-r_{1}\right)\left[u^{1}\left(r_{1}\right)+D\left(r_{1}\right) u^{1}(0)\right]-D(t) u^{1}\left(t-r_{1}\right) \\
& -\int_{r_{1}}^{t} A T(t-s) D(s) u^{1}\left(s-r_{1}\right) d s \\
& +\int_{r_{1}}^{t} T(t-s) f\left(s, u_{s}\right) d s, \quad t \in\left[r_{1}, 2 r_{1}\right],
\end{aligned}
$$

is completely continuous and has a fixed point $u^{2} \in C\left(\left[r_{1}-r, b\right] ; X\right)$.

To conclude the proof, we remark that the function obtained by pasting together $u^{1}(\cdot)$ and $u^{2}(\cdot)$ is a mild solution of (1.1)-(1.2) on $[-r, b]$. The proof is completed.

2.2. Existence of $S$-mild solutions. The results in the preceding section are proved by assuming a type of spatial regularity for the initial condition $\varphi$ and the function $s \rightarrow D(s) \varphi\left(s-r_{1}\right)$. In this section, we introduce another type of solution based on the assumption that $\varphi$ and $s \rightarrow D(s) \varphi\left(s-r_{1}\right)$ are 'regular' in the temporal variable.

Definition 2.5. A function $u \in C([-r+\sigma, \sigma+b] ; X), b>0, \sigma \in \mathbb{R}$, is called an $S$-mild solution of the neutral equation (2.1)-(2.2) if $u_{\sigma}=\varphi$, the function $s \rightarrow D(s) u\left(s-r_{1}\right)$ is differentiable almost everywhere on $[\sigma, \sigma+b], s \rightarrow(d / d s)$ $D(s) u\left(s-r_{1}\right) \in L^{1}([\sigma, \sigma+b], X)$ and

$$
u(t)=T(t-\sigma) \varphi(0)+\int_{\sigma}^{t} T(t-s)\left[-\frac{d}{d s} D(s) u\left(s-r_{1}\right)+f\left(s, u_{s}\right)\right] d s,
$$

for every $t \in[\sigma, \sigma+b]$.

In the rest of this section, $A_{W}$ is the generator of the semigroup $(W(t))_{t \geq 0}$ on $\mathcal{C}$ defined by $[W(t) \psi](\theta)=T(t+\theta) \psi(0)$ for $-t \leq \theta \leq 0$ and $[W(t) \psi](\theta)=\psi(t+\theta)$ for $-\infty<\theta<-t$.

The next result establishes the existence of an $S$-mild solution for (1.1)-(1.2).

Theorem 2.6. Assume that $\varphi \in D\left(A_{W}\right)$ and that the following conditions are satisfied.

(a) There exists $0<\alpha<1$ such that $D(\cdot) \in C^{1}\left([0, a] ; \mathcal{L}\left(X_{\alpha}, X\right)\right), g \in C^{2}\left(\left[0, r_{1}\right]\right.$; $\left.X_{\alpha}\right)$ and $\varphi(0) \in X_{1+\alpha}$.

(b) The function $f(\cdot)$ belongs to $C^{1}\left(\left[0, r_{1}\right] \times \mathcal{C}, X\right)$ and there exists $L_{f}>0$ such that

$$
\left\|f\left(t, \psi_{1}\right)-f\left(t, \psi_{2}\right)\right\| \leq L_{f}\left\|\psi_{1}-\psi_{2}\right\|_{\mathcal{C}}, \quad t \in\left[0, r_{1}\right], \psi_{i} \in \mathcal{C} .
$$

If $-d g(t) / d t_{\mid t=0}+f(0, \varphi)=0$, then there exists a unique $S$-mild solution of (1.1)-(1.2) on $\left[-r_{1}, b\right]$ for some $r_{1}<b \leq a$. 
PRoOF. Let $\Gamma_{1}: C\left(\left[-r, r_{1}\right] ; X\right) \rightarrow C\left(\left[-r, r_{1}\right] ; X\right)$ be the map defined by $(\Gamma u)_{0}=\varphi$ and

$$
\Gamma_{1} u(t)=T(t-\sigma) \varphi(0)+\int_{\sigma}^{t} T(t-s)\left[-\frac{d}{d s} g(s)+f\left(s, u_{s}\right)\right] d s, \quad t \in\left[0, r_{1}\right] .
$$

From condition (b) and the contraction mapping principle, it is easy to show that there exists a unique fixed point $u^{1}(\cdot)$ of $\Gamma(\cdot)$.

In order to show that $u^{1} \in C^{1}\left(\left[-r, r_{1}\right] ; X_{\alpha}\right)$, we introduce the delayed integral equation

$$
\begin{aligned}
& z(t)=A T(t) \varphi(0)-\int_{0}^{t} T(t-s) \frac{d^{2}}{d s^{2}} g(s) d s \\
& +\int_{0}^{t} T(t-s)\left[D_{1} f\left(s, u_{s}^{1}\right)+D_{2} f\left(s, u_{s}^{1}\right) z_{s}\right] d s, \quad t \in\left[0, r_{1}\right] \\
& z_{0}=A_{W} \varphi
\end{aligned}
$$

A standard argument using the contraction mapping principle allows us to prove that there exists a unique solution $z \in C\left(\left[-r, r_{1}\right] ; X\right)$ of (2.6)-(2.7). Moreover, arguing as in Henríquez [19], it follows that the functions $u^{1}(\cdot)$ and $t \rightarrow u_{t}^{1}$ are continuously differentiable on [0, $\left.r_{1}\right], d u^{1} d t=z$ and $d u_{t}^{1} d t=z_{t}$ on $\left[0, r_{1}\right]$.

Since $\varphi(0) \in X_{1+\alpha}$, from the above remark and the estimate

$$
\begin{aligned}
\left\|(-A)^{\alpha} \frac{d}{d t} u^{1}(t)\right\| \leq & \left\|A^{1+\alpha} T(t) \varphi(0)\right\|+\left\|\frac{d^{2} g}{d s^{2}}\right\|_{C\left(\left[0, r_{1}\right] ; X_{\alpha}\right)} \int_{0}^{t} \frac{C_{1-\alpha}}{(t-s)^{1-\alpha}} d s \\
& +\sup _{\tau \in\left[0, r_{1}\right]}\left\|D_{1} f\left(\tau, u_{\tau}^{1}\right)+D_{2} f\left(\tau, u_{\tau}^{1}\right) z_{\tau}\right\| \int_{0}^{t} \frac{C_{\alpha}}{(t-s)^{\alpha}} d s \\
\leq C+ & C_{1-\alpha}\left\|\frac{d^{2} g}{d s^{2}}\right\|_{C\left(\left[0, r_{1}\right] ; X_{\alpha}\right)} \frac{r_{1}^{\alpha}}{\alpha} \\
& +C_{\alpha} \sup _{\tau \in\left[0, r_{1}\right]}\left\|D_{1} f\left(\tau, u_{\tau}^{1}\right)+D_{2} f\left(\tau, u_{\tau}^{1}\right) z_{\tau}\right\| \frac{r_{1}^{1-\alpha}}{1-\alpha},
\end{aligned}
$$

where $C$ is a constant independent of $t$, we infer that $d u^{1}(t) d t \in X_{\alpha}$ for all $t \in\left[0, r_{1}\right], u^{1} \in C^{1}\left(\left[0, r_{1}\right] ; X_{\alpha}\right)$ and the function $t \rightarrow D(t) u^{1}\left(t-r_{1}\right)$ belongs to $C^{1}\left(\left[r_{1}, 2 r_{1}\right] ; X\right)$.

From the properties of $f(\cdot)$, there are positive constants $C, b$ and $L$ such that

$$
\left\|f\left(t, \psi_{1}\right)-f\left(t, \psi_{2}\right)\right\| \leq L\left\|\psi_{1}-\psi_{2}\right\|_{\mathcal{C}},
$$

and $\left\|f\left(t, \psi_{1}\right)\right\| \leq C$ for all $\left(t, \psi_{i}\right) \in\left[r_{1}, r_{1}+b\right] \times B_{b}\left(u_{r_{1}}^{1}, \mathcal{C}\right)$.

Let $0<b_{1}<b$ be such that $M L b_{1}<1$ and

$$
\begin{gathered}
\sup _{s \in\left[r_{1}, r_{1}+b_{1}\right]}\left\|y_{s}-\left(u^{1}\right) r_{1}\right\|_{\mathcal{C}} \leq \frac{b}{2}, \\
M \sup _{s \in\left[r_{1}, r_{1}+b_{1}\right]}\left\|\frac{d}{d s} D(s) u^{1}\left(s-r_{1}\right)\right\| b_{1}+M C b_{1} \leq \frac{b}{2} .
\end{gathered}
$$


Let $\Lambda$ be the space

$$
\Lambda=\left\{u \in C\left(\left[r_{1}-r, r_{1}+b_{1}\right] ; X\right): u_{r_{1}}=\left(u^{1}\right)_{r_{1}},\|u-y\|_{C\left(\left[r_{1}, r_{1}+b_{1}\right] ; X\right)} \leq \frac{b}{2}\right\}
$$

endowed with the supremum norm, and $\Gamma_{2}: \Lambda \rightarrow C\left(\left[r_{1}-r, r_{1}+b_{1}\right] ; X\right)$ be the map defined by $\left(\Gamma_{2} u\right)_{r_{1}}=\left(u^{1}\right)_{r_{1}}$ and

$$
\Gamma_{2} u(t)=T\left(t-r_{1}\right) u^{1}(0)+\int_{r_{1}}^{t} T(t-s)\left[-\frac{d}{d s} D(s) u^{1}\left(s-r_{1}\right)+f\left(s, u_{s}\right)\right] d s,
$$

for $t \in\left[r_{1}, r_{1}+b_{1}\right]$.

We next prove that $\Gamma_{2}$ is a contraction from $\Lambda$ into $\Lambda$. For $u \in \Lambda$ and $t \in$ $\left[r_{1}, r_{1}+b_{1}\right]$ we see that

$$
\begin{aligned}
\left\|u_{t}-\left(u^{1}\right)_{r_{1}}\right\|_{\mathcal{C}} & \leq\left\|u_{t}-y_{t}\right\|_{\mathcal{C}}+\left\|y_{t}-\left(u^{1}\right)_{r_{1}}\right\|_{\mathcal{C}} \\
& \leq\|u-y\|_{C\left(\left[r_{1}, r_{1}+b_{1}\right] ; X\right)}+\frac{b}{2} \\
& \leq b,
\end{aligned}
$$

so that $u_{t} \in B_{b}\left(u_{r_{1}}^{1}, \mathcal{C}\right)$. Consequently, $\left\|f\left(s, u_{s}\right)\right\| \leq C$ for all $s \in\left[r_{1}, r_{1}+b_{1}\right]$ and

$$
\left\|\Gamma_{2} u(t)-y(t)\right\| \leq \sup _{s \in\left[r_{1}, r_{1}+b_{1}\right]}\left\|\frac{d}{d s} D(s) u^{1}\left(s-r_{1}\right)\right\| b_{1}+M C b_{1} \leq \frac{b}{2},
$$

for all $t \in\left[r_{1}, r_{1}+b_{1}\right]$. Thus, $\Gamma_{2} u \in \Lambda$ and $\Gamma_{2} \Lambda \subset \Lambda$. Moreover, for $u, v \in \Lambda$ we find that

$$
\left\|\Gamma_{2} u-\Gamma_{2} v\right\|_{C\left(\left[r_{1}, r_{1}+b_{1}\right] ; X\right)} \leq M L b_{1}\|u-v\|_{C\left(\left[r_{1}, r_{1}+b_{1}\right] ; X\right)},
$$

which proves that $\Gamma_{2}(\cdot)$ is a contraction on $\Lambda$ and that there exists a unique fixed point $u^{2}(\cdot)$ of $\Gamma_{2}(\cdot)$.

Finally, by pasting together the functions $u^{i}(\cdot)$ we obtain an $S$-mild solution of (1.1)-(1.2) on $[0, b]$. The proof is now complete.

To conclude this section, we consider the case in which $\mathcal{D} \supset D(A)$. The proofs of our next results follow with minor modifications from the proof of Theorem 2.3 and Theorem 2.6. We only give a sketch of the proofs for completeness.

TheOREM 2.7. Assume that $f$ satisfies condition (c) of Theorem 2.3 and that there are $\sigma_{1} \in(0,1)$ and $1>\sigma_{2}>\sigma_{3}>0$ such that $f \in C\left(\left[0, r_{1}\right] \times C\left([-r, 0] ; X_{\sigma_{2}}\right) ; X_{\sigma_{3}}\right)$ and $g \in C\left([0, a] ; X_{1+\sigma_{1}}\right)$. Suppose that there is a Banach space $\left(Y,\|\cdot\|_{Y}\right) \hookrightarrow$ $(X,\|\cdot\|)$ and $0<\sigma<\min \left\{\sigma_{1}, \sigma_{3}\right\}$ such that $D(\cdot) \in C\left([0, a] ; \mathcal{L}\left(X_{1+\sigma}, Y\right)\right), \varphi(0) \in$ $X_{1+\sigma}$ and $A T(\cdot) \in L^{1}([0, a], \mathcal{L}(Y, X))$. Then there exists a unique mild solution of (1.1)-(1.2) on $\left[-r, 2 r_{1}\right]$. 
PROOF. Let $\Gamma_{1}(\cdot)$ and $u^{1}(\cdot)$ be as in the proof of Theorem 2.3. Proceeding as in that proof, we can prove that $u^{1} \in C\left(\left[0, r_{1}\right] ; X_{\theta}\right)$ for every $\theta \in(0,1)$ which in turn implies that the function $t \rightarrow f\left(t, u_{t}^{1}\right)$ belongs to $C\left(\left[0, r_{1}\right] ; X_{\sigma_{3}}\right)$. Now, from the estimate

$$
\begin{aligned}
\left\|(-A)^{1+\sigma} u^{1}(t)\right\| \leq\left\|(-A)^{1+\sigma} T(t)[\varphi(0)+g(0)]-(-A)^{1+\sigma} g(t)\right\| \\
\quad \times \int_{r_{1}}^{t}\left\|A^{1-\left(\sigma_{1}-\sigma\right)} T(t-s)\right\|\left\|(-A)^{1+\sigma_{1}} g(s)\right\| d s \\
\quad+\int_{r_{1}}^{t}\left\|(-A)^{1-\left(\sigma_{3}-\sigma\right)} T(t-s)\right\|\left\|(-A)^{\sigma_{3}} f\left(s, u_{s}^{1}\right)\right\| d s \\
\leq C+C_{1-\left(\sigma_{1}-\sigma\right)\|g\|_{C\left([0, a] ; X_{1+\sigma_{1}}\right)} \frac{r_{1}^{\sigma_{1}-\sigma}}{\sigma-\sigma}} \\
+C_{1-\left(\sigma_{3}-\sigma\right)} \sup _{\tau \in\left[0, r_{1}\right]}\left\|(-A)^{\sigma_{3}} f\left(\tau, u_{\tau}^{1}\right)\right\| \frac{r_{1}^{\sigma_{3}-\sigma}}{\sigma_{3}-\sigma},
\end{aligned}
$$

we infer that $u^{1} \in C\left(\left[0, r_{1}\right] ; X_{1+\sigma}\right)$ and, as a consequence, that the function $t \rightarrow$ $D(t) u^{1}\left(t-r_{1}\right)$ belongs to $C\left(\left[r_{1}, 2 r_{1}\right] ; Y\right)$. Now the proof can be completed by arguing as in the last part of the proof of Theorem 2.3. We omit the additional details.

For the $S$-mild solution case we have the following result.

Theorem 2.8. Assume that $D(\cdot) \in C^{1}\left([0, a] ; \mathcal{L}\left(X_{1}, X\right)\right), \varphi(0) \in X_{2}, \varphi \in D\left(A_{W}\right)$ and $f(\cdot)$ satisfies condition $(c)$ of Theorem 2.6. Suppose that there are numbers $\sigma_{1} \in(0,1)$ and $0<\sigma_{3}<\sigma_{2}<1$ such that $g \in C^{2}\left(\left[0, r_{1}\right] ; X_{\sigma_{1}}\right)$ and $f \in C^{1}\left(\left[0, r_{1}\right] \times\right.$ $\left.C\left([-r, 0] ; X_{\sigma_{2}}\right), X_{\sigma_{3}}\right)$. If $f(0, \varphi)-d g(t) / d t_{t_{t=0}}=0$, then there exists a unique $S$-mild solution of (1.1)-(1.2) on $\left[-r_{1}, b\right]$ for some $r_{1}<b \leq a$.

ProOf. Let $\Gamma_{1}(\cdot), u^{1}(\cdot)$ and $z(\cdot)$ be as in the proof of Theorem 2.6. Arguing as in that proof, we can show that $u^{1} \in C^{1}\left(\left[0, r_{1}\right] ; X_{\theta}\right)$ for all $\theta \in(0,1)$, which implies that the function $t \rightarrow d f\left(t, u_{t}^{1}\right) d t$ belongs to $C\left(\left[0, r_{1}\right] ; X_{\sigma_{3}}\right)$. Now using the fact that $\varphi(0) \in X_{2}, z=d u^{1} d t$ is the solution of (2.6)-(2.7) and $d u_{t}^{1} d t=z_{t}$, we obtain

$$
\begin{aligned}
\left\|A \frac{d}{d t} u^{1}(t)\right\| & \\
\leq & \left\|A^{2} T(t) \varphi(0)\right\|+\int_{0}^{t}\left\|(-A)^{1-\sigma_{1}} T(t-s)(-A)^{\sigma_{1}} \frac{d^{2}}{d s^{2}} g(s)\right\| d s \\
& +\int_{0}^{t}\left\|(-A)^{1-\sigma_{3}} T(t-s)(-A)^{\sigma_{3}}\left(D_{1} f\left(s, u_{s}^{1}\right)+D_{2} f\left(s, u_{s}^{1}\right) z_{s}\right)\right\| d s \\
\leq & C+C_{1-\sigma_{1}}\left\|\frac{d^{2}}{d s^{2}} g(s)\right\|_{C\left(\left[0, r_{1}\right] ; X_{\sigma_{1}}\right)} \frac{r_{1}^{\sigma_{1}}}{\sigma_{1}}+C_{1-\sigma_{3}}\left\|\frac{d}{d s} f\left(s, u_{s}^{1}\right)\right\|_{C\left(\left[0, r_{1}\right] ; X_{\sigma_{3}}\right)} \frac{r_{1}^{\sigma_{3}}}{\sigma_{3}},
\end{aligned}
$$

which shows that $u^{1} \in C^{1}\left(\left[0, r_{1}\right] ; X_{1}\right)$ and $t \rightarrow D(t) u^{1}\left(t-r_{1}\right) \in C^{1}\left(\left[r_{1}, 2 r_{1}\right] ; Y\right)$ since $D(\cdot) \in C^{1}\left([0, a] ; \mathcal{L}\left(X_{1}, Y\right)\right)$. Arguing now as in the last part of the proof of 
Theorem 2.6, we can establish the existence of an $S$-mild solution on $\left[-r_{1}, b\right]$ for some $r_{1}<b \leq a$.

\section{An application}

In this section we apply our abstract results to study the existence of mild and $S$-mild solutions for a concrete partial neutral differential equation. Specifically, we consider the 'degenerate' neutral system

$$
\begin{gathered}
\frac{\partial}{\partial t}\left(u(t, \xi)+\alpha(t) \frac{\partial^{i}}{\partial \xi^{i}} u\left(t-r_{1}, \xi\right)\right) \\
=\frac{\partial^{2}}{\partial \xi^{2}} u(t, \xi)+\sum_{i=1}^{m} \beta_{i}(t) u\left(t-r_{i}^{1}, \xi\right)+\alpha_{1}(t) \int_{t-r}^{t} \eta(s-t) u(s, \xi) d s \\
u(t, 0)=u(t, \pi)=0, \\
u(\theta, \xi)=\varphi(\theta, \xi), \quad \theta \in[-r, 0], \xi \in[0, \pi]
\end{gathered}
$$

for $i=1,2$, where $\alpha, \alpha_{1}, \beta_{i} \in C([0, a] ; \mathbb{R}), \eta \in L^{2}([0, a]), r, r_{1}, r_{i}^{1}, i=1, \ldots, m$, are real numbers and $0<r_{1}<r$.

To treat the system (3.1)-(3.3) under the abstract framework used in the Section 2, we need to introduce some technicalities. In what follows, $X=L^{2}([0, \pi]), \mathcal{C}=$ $C([-r, 0] ; X), D(A):=\left\{x \in X: x^{\prime \prime} \in X, x(0)=x(\pi)=0\right\}$ and $A: D(A) \subset X \rightarrow X$ is the operator defined by $A x=x^{\prime \prime}$. It is well known that $A$ is the infinitesimal generator of an analytic semigroup $(T(t))_{t \geq 0}$ on $X$. Furthermore, $A$ has discrete spectrum with eigenvalues $-n^{2}, n \in \mathbb{N}$, and corresponding normalized eigenfunctions given by $z_{n}(\xi)=(2 / \pi)^{1 / 2} \sin (n \xi)$, the set of functions $\left\{z_{n}: n \in \mathbb{N}\right\}$ is an orthonormal basis of $X$ and $T(t) x=\sum_{n=1}^{\infty} e^{-n^{2} t}\left\langle x, z_{n}\right\rangle z_{n}$ for $x \in X$. It follows from this representation that $(T(t))_{t \geq 0}$ is a compact semigroup on $X$. Let us consider the cases $i=1$ and $i=2$ separately.

3.1. The case $i=1$. Let $\alpha \in(1 / 2,1)$ and $D(t): X_{\alpha} \rightarrow X, f:[0, a] \times \mathcal{C} \rightarrow X$ be the functions defined by $D(t) x(\xi)=\alpha(t) x^{\prime}(\xi)$ and

$$
f(t, \psi)(\xi)=\sum_{i=1}^{m} \beta_{i}(t) \psi\left(-r_{i}^{1}, \xi\right)+\alpha_{1}(t) \int_{-r}^{0} \eta(s) \psi(s, \xi) d s .
$$

It is easy to see that $D(\cdot) \in C\left([0, a] ; \mathcal{L}\left(X_{\alpha}, X_{\alpha-\frac{1}{2}}\right)\right), A T(\cdot) \in C\left([0, a] ; \mathcal{L}\left(X_{\alpha-\frac{1}{2}}, X\right)\right)$, $f \in C([0, a] ; \mathcal{L}(\mathcal{C}, X))$ and $\|f(t, \cdot)\|_{\mathcal{L}(\mathcal{C}, X)} \leq \sum_{i=1}^{m}\left\|\beta_{i}\right\|_{C([0, b] ; \mathbb{R})}+\left\|\alpha_{1}\right\|_{C([0, b] ; \mathbb{R})} r^{1 / 2}\|\eta\|_{L^{2}([0, b] ; \mathbb{R})}, \quad t \in[0, a]$.

Under these conditions, it is possible to describe the system (3.1)-(3.3) in the abstract form (1.1)-(1.2). In the next result, which is a consequence of Theorems 2.3 
and 2.6, we say that a function $u \in C([-r, b] ; X)$ is a mild solution (an $S$-mild solution) of (3.1)-(3.3) on $[0, b]$ if $u(\cdot)$ is a mild solution (an $S$-mild solution) of the associated abstract system (1.1)-(1.2) on $[0, b]$.

PROPOSITION 3.1. The following assertions hold.

(a) If $\varphi \in C\left([-r, 0] ; X_{\alpha+\mu}\right)$ for some $0<\mu<1-\alpha$, then there exists a unique mild solution of (3.1)-(3.3) on $\left[-r, 2 r_{1}\right]$.

(b) Assume that $\alpha \in C^{2}([0, a] ; \mathbb{R}), \varphi \in C^{2}\left([-r, 0] ; X_{\alpha}\right), \varphi(0) \in X_{1+\alpha}, \varphi \in D\left(A_{W}\right)$, the functions $\alpha_{1}, \beta_{i}$ are of class $C^{1}$ and

$$
\begin{gathered}
-\frac{d}{d t}\left[\alpha(t) \varphi\left(t-r_{1}, \xi\right)\right]_{t=0}+\sum_{i=1}^{m} \beta_{i}(0) \varphi\left(-r_{i}^{1}, \xi\right) \\
+\alpha_{1}(0) \int_{-r}^{0} \eta(s) \varphi(s, \xi) d s=0
\end{gathered}
$$

almost everywhere for $\xi \in[0, \pi]$. Then there exists a unique $S$-mild solution of (3.1)-(3.3) on $\left[-r_{1}, b\right]$ for some $r_{1}<b \leq a$.

3.2. The case $\boldsymbol{i}=2$. In this case we consider the maps $D(t): D(A) \subset X \rightarrow X$ given by $D(t) x=\alpha(t) x^{\prime \prime}$. As in the above case, we say that $u \in C([-r, b] ; X)$ is a mild solution (an $S$-mild solution) of (3.1)-(3.3) on $[0, b]$ if $u(\cdot)$ is a mild solution (an $S$-mild solution) of the associated neutral system (1.1)-(1.2) on $[0, b]$. The next result follows from Theorems 2.7 and 2.8 .

PROPOSITION 3.2. The following assertions hold.

(a) Assume that $\alpha \in C([0, a] ; \mathbb{R})$ and that there is $\sigma_{1}>0$ such that $\varphi \in$ $C\left([0, a] ; X_{2+\sigma_{1}}\right)$. Then there exists a unique mild solution of (3.1)-(3.3) on $\left[-r, 2 r_{1}\right]$.

(b) Suppose that $\varphi \in D\left(A_{W}\right), \alpha \in C^{2}([0, a] ; \mathbb{R})$ and $\varphi \in C^{2}\left([-r, 0] ; X_{1+\sigma_{1}}\right)$ for some $\sigma_{1}>0$. If $\varphi \in D\left(A_{W}\right), \varphi(0) \in X_{2}$ and (3.4) is satisfied, then there exists a unique $S$-mild solution of (1.1)-(1.2) on $\left[-r_{1}, b\right]$ for some $r_{1}<b \leq a$.

\section{References}

[1] M. Adimy and K. Ezzinbi, 'A class of linear partial neutral functional-differential equations with nondense domain', J. Differential Equations 147(2) (1998), 285-332.

[2] K. Balachandran and R. Sakthivel, 'Existence of solutions of neutral functional integrodifferential equation in Banach spaces', Proc. Indian Acad. Sci. Math. Sci. 109(3) (1999), 325-332.

[3] K. Balachandran, G. Shija and J. H. Kim, 'Existence of solutions of nonlinear abstract neutral integrodifferential equations', Comput. Math. Appl. 48(10-11) (2004), 1403-1414.

[4] M. Benchohra, J. Henderson and S. K. Ntouyas, 'Existence results for impulsive multivalued semilinear neutral functional differential inclusions in Banach spaces', J. Math. Anal. Appl. 263(2) (2001), 763-780.

[5] M. Benchohra and S. K. Ntouyas, 'Nonlocal Cauchy problems for neutral functional differential and integrodifferential inclusions in Banach spaces', J. Math. Anal. Appl. 258(2) (2001), 573-590.

[6] P. Cannarsa and D. Sforza, 'Global solutions of abstract semilinear parabolic equations with memory terms', Nonlinear Differ. Equat. Appl. 10(4) (2003), 399-430. 
[7] P. Clément and J. A. Nohel, 'Asymptotic behavior of solutions of nonlinear Volterra equations with completely positive kernels', SIAM J. Math. Anal. 12(4) (1981), 514-535.

[8] P. Clément and J. Prüss, 'Global existence for a semilinear parabolic Volterra equation', Math. Z. 209(1) (1992), 17-26.

[9] R. Datko, 'Linear autonomous neutral differential equations in a Banach space', J. Differential Equations 25(2) (1977), 258-274.

[10] J. P. Dauer and K. Balachandran, 'Existence of solutions of nonlinear neutral integrodifferential equations in Banach spaces', J. Math. Anal. Appl. 251(1) (2000), 93-105.

[11] H. Fang and J. Li, 'On the existence of periodic solutions of a neutral delay model of single-species population growth', J. Math. Anal. Appl. 259(1) (2001), 8-17.

[12] H. I. Freedman and Y. Kuang, 'Some global qualitative analyses of a single species neutral delay differential population model. Second Geoffrey J. Butler Memorial Conference in Differential Equations and Mathematical Biology (Edmonton, AB, 1992)', Rocky Mountain J. Math. 25(1) (1995), 201-215.

[13] A. Granas and J. Dugundji, Fixed Point Theory (Springer, New York, 2003).

[14] M. E. Gurtin and A. C. Pipkin, 'A general theory of heat conduction with finite wave speed', Arch. Ration. Mech. Anal. 31 (1968), 113-126.

[15] J. K. Hale and S. M. Verduyn Lunel, Introduction to Functional-Differential Equations, Applied Mathematical Sciences, 99 (Springer, New York, 1993).

[16] E. Hernández, 'Existence results for partial neutral integrodifferential equations with unbounded delay', J. Math. Anal. Appl. 292(1) (2004), 194-210.

[17] E. Hernández and H. Henríquez, 'Existence results for partial neutral functional differential equation with unbounded delay', J. Math. Anal. Appl. 221(2) (1998), 452-475.

[18] E. Hernández and D. O'Regan, 'Existence results for abstract partial neutral differential equations', Proc. Amer. Math. Soc. 137 (2009), 3309-3318.

[19] H. R. Henríquez, 'Regularity of solutions of abstract retarded functional-differential equations with unbounded delay', Nonlinear Anal. 28(3) (1997), 513-531.

[20] Y. Kuang, 'Qualitative analysis of one- or two-species neutral delay population models', SIAM J. Math. Anal. 23(1) (1992), 181-200.

[21] Q. Li, J. Cao and S. Wan, 'Positive periodic solution for a neutral delay model in population', J. Biomath. 13(4) (1998), 435-438.

[22] A. Lunardi, 'On the linear heat equation with fading memory', SIAM J. Math. Anal. 21(5) (1990), 1213-1224.

[23] J. W. Nunziato, 'On heat conduction in materials with memory', Quart. Appl. Math. 29 (1971), 187-204.

[24] A. Pazy, Semigroups of Linear Operators and Applications to Partial Differential Equations, Applied Mathematical Sciences, 44 (Springer, New York, 1983).

EDUARDO HERNÁNDEZ, Departamento de Matemática, I.C.M.C. Universidade de São Paulo, Caixa Postal 668, 13560-970 São Carlos SP, Brazil e-mail: lalohm@icmc.usp.br

KRISHNAN BALACHANDRAN, Department of Mathematics, Bharathiar University, Coimbatore 641046, India e-mail:kbkb1956@yahoo.com 